REVISTA DE DERECHO UNED, NÚM. 18, 2016

\title{
LÍMITES ÉTICOS Y JURÍDICOS DE LA INVESTIGACIÓN BIOMÉDICA
}

\section{ETHICAL AND LEGAL LIMITS OF BIOMEDICAL RESEARCH}

\author{
Dr. Francisco Rivas García \\ Doctorando Ciencias Sociales y Derecho. \\ Facultad de Derecho. UNED
}

Resumen: El desarrollo científico en el campo de la Biomedicina ha generado una nueva forma de aproximarse al estudio del ser humano mediante técnicas de investigación que incluyen, entre otras, la ingeniería genética, la reproducción humana asistida y los trasplantes de órganos. El presente trabajo permite situar un marco general sobre los distintos aspectos generales que regulan e inciden en la investigación biomédica.

Abstract: The advance of science has created a new way of approaching the study of the human being through biomedical research techniques and their protection, with focused issues, genetic engineering, assisted human reproduction, mechanisms of artificial fertilization, transplants organ and, in general, in the various processes in the context of biomedical research. The present review lets you put a framework on various general aspects that regulate and influence biomedical research in general, and the various existing to it, combining the legislative limits, and ethical aspect.

Palabras clave: Investigación biomédica. Bioderecho. Legislación. Valores ético-jurídicos.

Keywords: biomedical research. Biolaw. Legislation. Ethical and legal values. 
Recepción original: 26/02/2016

Aceptación original: 6/04/2016

Sumario: I. Introducción. II. Bioética y derecho. II.1 Bioética y bioderecho. II.2 Dignidad humana e investigación biomédica. II.3 Bioderecho. III. Investigación biomédica en el marco de la Bioética y Bioderecho. IV. Legislación en investigación biomédica. IV.1 Legislación internacional. IV.2 Legislación Europea. IV.3 Legislación Española. V. Ley de Investigación Biomédica como límite en el ordenamiento jurídico español. VI. Conclusiones.

\section{INTRODUCCIÓN}

El objetivo fundamental de cualquier investigación biomédica es profundizar en el conocimiento de los mecanismos moleculares, bioquímicos, celulares, genéticos, fisiopatológicos y epidemiológicos de las enfermedades para identificar los problemas de salud, y poder establecer las estrategias e intervenciones adecuadas para su prevención y tratamiento ${ }^{1}$.

Son numerosas las disciplinas científicas (medicina, biología, bioquímica y genética) que aúnan esfuerzos conjuntos con otras ciencias de la salud (epidemiología, sociología y economía) para aumentar el conocimiento sobre el ser humano, así como el desarrollo de técnicas y/o procedimientos en el campo de la biomedicina.

Los recientes avances en el campo de la biomedicina están siendo objeto de estudio por parte de la Filosofía del Derecho, que engloba numerosos estudios que requieren de la Bioética para su comprensión e interpretación. Por todo ello, el Derecho no puede ni debe quedar apartado de la investigación biomédica sino que se necesita para poder ofrecer una respuesta adecuada y válida ante los actuales interrogantes y los futuros retos que implicarán nuevos descubrimientos científicos.

El rápido avance de las técnicas de investigación en materia biomédica genera ciertas dudas, de ahí, que se hace necesario reflexionar sobre los límites a definir en el campo de la biomedicina para establecer una separación entre todo lo que se debe hacer y lo que desde el punto de vista técnico es posible realizar.

${ }^{1}$ CA MÍ, J., «Mapa bibliométrico de España 1994-2002: Biomedicina y Ciencias de la Salud», Med. Clin. (Barc), 2005; 124(3), 93-101. 


\section{BIOÉTICA Y BIODERECHO}

\section{II.1 Bioética}

La Bioética es una disciplina que estudia, analiza y resuelve todas aquellas cuestiones éticas referidas a la protección de la vida. Su origen surge como consecuencia de una serie de circunstancias históricas basadas en el desarrollo de investigaciones en el campo de la biología y medicina que carecían de las básicas consideraciones sobre la protección de la vida y dignidad de las personas. ${ }^{2}$

Comprender los fundamentos de la bioética exige conocer el Informe Belmont ${ }^{3}$, que establece los principios y guías éticas para la protección de las personas sujetas a investigación. Los principios de beneficencia, autonomía, justicia y maleficencia, que se detallarán más adelante, constituyen la base útil para la aplicación teóricopráctica de las decisiones de la bioética. Sin duda, estos principios constituyen la herramienta de decisión del modelo anglosajón y europeo siendo la protección de la dignidad humana ${ }^{4}$ el pilar fundamental a considerar. A continuación se describen, a rasgos generales, cada uno de los principios:

\section{Principio de No-Maleficencia}

En este principio se establece genéricamente que no se debe causar ningún daño de tipo físico, psíquico, moral y/o económico a las personas sometidas a cualquier investigación. Existiría maleficencia cuando la relación beneficio-riesgo de una intervención pueda considerarse a priori como desfavorable .

\section{Principio de Justicia}

Fue definido en el Informe Belmont como una distribución equitativa de las cargas y los beneficios de la investigación entre todos los

2 ACEVEDO PEREZ, A., "Aspectos éticos en la investigación científica», Ciencia y Enfermería., jun. 2002, vol.8, no.1, p. 15-18.

${ }^{3}$ The National Commission for the Protection of Human Subjects of Biomedical and Behavioral Research. Belmont Report. [acceso: 27 de octubre de 2011]. Disponible en: http://ohsr.od.nih.gov/guidelines/ belmont.html

${ }^{4}$ DE LA TORRE, J. (ed.), Dignidad humana y bioética, UPCO, Madrid 2008.

${ }^{5}$ GRACIA, D., «La deliberación moral: el método de la ética clínica». Med Clin (Barc), 2001; 117:16-17 
individuos participantes en el problema que se investiga. Por tanto, se debe garantizar una selección equitativa de las personas que participen en investigación ${ }^{6}$.

\section{Principio de Autonomía}

El principio se enmarca dentro del derecho a la libertad de los individuos y exige el respeto por los criterios, consideraciones, preferencias y acciones de las personas autónomas. Se define como persona autónoma aquella que tiene la capacidad de deliberar sobre sus fines personales, y de obrar en consecuencia ${ }^{7}$.

\section{Principio de Beneficencia}

Atendiendo a lo descrito en el Informe Belmont «se trata a las personas de manera ética no sólo respetando sus decisiones y protegiéndolas de daño, sino garantizando su bienestar». Por tanto, el término beneficencia se refiere a todos los actos de bondad y de caridad que pueden y deben incorporarse potencialmente a la investigación biomédica ${ }^{8}$.

\section{II.2 Dignidad humana e investigación biomédica}

La bioética considera la dignidad humana como el derecho básico a proteger en cualquier investigación. En este sentido, quizás existan dudas sobre las circunstancias que pueden afectar a la dignidad humana, para ello, la Declaración Universal de los Derechos Humanos expone que se atenta a la dignidad humana cuando se viola la libertad de la persona, se somete a estado de esclavitud, se destruyen embriones engendrados, se comercia con tejidos humanos, se generan tratos degradantes y no se respeta la vida privada e intimidad de las personas ${ }^{9}$.

${ }^{6}$ DE ABAJO, FJ., «La Declaración de Helsinki VI: una revisión necesaria, pero ¿suficiente?», Rev Esp Salud Pública 2001; 75: 407-420

${ }^{7}$ JÚDEZ, J., «La confidencialidad en la práctica clínica: historia clínica y gestión de la información», Med Clin (Barc) 2002; 118:18-37.

${ }^{8}$ RUIZ-MATEOS, AM., «El 'ethos' médico ante la experimentación en seres humanos», Moralia, 17-18, 1983, pp. 505ss

${ }^{9}$ Instrumento de Ratificación del Convenio para la Protección de los Derechos Humanos y de las Libertades Fundamentales, hecho en Roma el 4 de noviembre de 1950, y enmendado por los Protocolos adicionales números 3 y 5 , de 6 de mayo de 
Dignidad es un término que presenta diversas dificultades en su concepción pero no solo se refiere al ámbito ético sino que es un valor que está unido, de manera indisoluble, a las propias personas y por tanto, es la misma para todos los seres humanos con independencia de sus actos éticos ${ }^{10}$. El concepto de dignidad humana viene reconocido y garantizado en las principales declaraciones y pactos internacionales que rigen cualquier investigación biomédica.

Atendiendo a la importancia de la dignidad, como derecho fundamental a proteger, Ezekiel Emmanuel estableció una serie de requisitos que debe cumplir una investigación biomédica para que contribuya al bien social y garantice la protección, dignidad y respeto de las personas ${ }^{11}$. Básicamente, lo enunciado por Ezekiel resalta la importancia del valor y mejora del bienestar como fundamento básico de cualquier investigación biomédica, junto con el buen diseño y validez de la metodología científica aplicada. Otros requisitos se centran en la selección equitativa de las personas participantes, priorizar el bienestar ante otros intereses distintos de investigación y potenciar la importancia del consentimiento informado como un documento básico que garantiza la adecuada información y aceptación de las condiciones de cualquier investigación biomédica ${ }^{12}$, todo ello para que las personas participantes puedan decidir libremente.

\section{II.3 Bioderecho}

El Bioderecho se puede definir como una parte del Derecho que tiene por objeto el estudio y regulación de las nuevas situaciones biomédicas desde el respeto de la dignidad y los derechos del ser huma$n^{13}$. Los fundamentos del Bioderecho tienen como base la dignidad humana y los derechos inherentes al ser humano, los cuales constitu-

1963 y 20 de enero de 1966, respectivamente. BOE núm. 243, de 10 de octubre de 1979 , p. 23564.

${ }^{10}$ ANDORNO, R., Bioética y dignidad de la persona, Tecnos, 1998, pp.21ss

${ }^{11}$ EMMANUEL, E., "¿Qué hace que la investigación clínica sea ética? Siete requisitos éticos» en Pautas éticas de investigación en sujetos humanos: nuevas perspectivas, Programa regional de bioética OPS, OMS, 2003.

${ }^{12}$ FEITO, L., «Principios vs consecuencias», En: Álvarez, JC, ed, principios y aplicaciones de la bioética. Asociación de Bioética Fundamental y Clínica, Madrid 2005, p. 125.

${ }^{13}$ MARCOS DEL CANO, AM., "La biojurídica en España», Rivista internazionale di Filosofia del Diritto, 1984, gennaio-marzo, LXXI, pp. 124. 
yen una herramienta que establece el marco jurídico necesario para el desarrollo de la investigación biomédica ${ }^{14}$.

Resulta complejo considerar el ámbito del Bioderecho sin Bioética y viceversa, por lo que ambas disciplinas deben trabajar conjuntamente; así, la Bioética aportará una reflexión ética y multidisciplinar mientras que el Bioderecho dispondrá de los instrumentos complementarios ${ }^{15}$. No obstante, los principios reguladores y/o imposición de limitaciones en materia de Bioderecho aseguran el pluralismo ideológico y la uniformidad de principios regulados jurídicamente ${ }^{16}$ entre los que se encuentran garantizar la libertad de producción científica, proteger los resultados de las investigaciones, promover la investigación científica y limitar las aplicaciones de las técnicas de investigación biomédica si estas afectan a los derechos humanos relacionados con el bien físico y moral ${ }^{17}$.

\section{INVESTIGACIÓN BIOMÉDICA EN EL MARCO DE LA BIOÉTICA Y BIODERECHO}

Bioética y Bioderecho se integran y complementan en la libertad de investigación, derecho fundamental, tal y como consagra la Constitución Española (CE) en su artículo 20, que debe respetarse en sus distintas variantes, entre las que se encuentra la investigación biomédica. Aparte de lo descrito en el texto constitucional español, el derecho de libertad de investigación está reconocido en la Declaración Universal de los Derechos Humanos (artículos 18 y 19), Declaración Universal de Bioética y Derechos Humanos (artículo 2) y el Pacto Internacional de Derechos Civiles y Políticos (artículo 19.2) ${ }^{18}$, Carta de Derechos Fundamentales de la Unión Europea (artículo 13) ${ }^{19}$ y Convenio de Protección de Derechos Humanos y Dignidad (artículo 15) ${ }^{20}$.

${ }^{14}$ JUNQUERA DE ESTEFANI, R., «Los Derechos Humanos: criterios referenciales para la Bioética», Moralia, 2005, 28(105): 7-32.

${ }^{15}$ VID D’AGOSTINO, F., "Ética y derecho en bioética», en Bioética. Estudios de Filosofía del Derecho, op. cit., pág. 68.

${ }^{16}$ OLLERO, A., "De la bioética a la biopolítica», en Bioderecho. Entre la vida y la muerte, Thomson Aranzadi, Pamplona, 2006, p. 65.

${ }^{17}$ APARISI, A., «Bioética, Bioderecho y Biojurídica, Reflexiones desde la Filosofía del Derecho», Anuario de Filosofía del Derecho, 24, 2007, p. 63.

${ }^{18}$ Instrumento de Ratificación de España del Pacto Internacional de Derechos Civiles y Políticos, hecho en Nueva York el 19 de diciembre de 1966. BOE núm. 103, de 30 de abril de 1977, p. 9337.

${ }^{19}$ Carta de los Derechos Fundamentales de la Unión Europea. DOUE, núm. 83, de 30 de marzo de 2010, p. 389.

${ }^{20}$ INSTRUMENTO de Ratificación del Convenio para la protección de los derechos humanos y la dignidad del ser humano con respecto a las aplicaciones de la 
Por todo ello, atendiendo a las normas legislativas, este derecho de libertad de investigación debe ser respetado y protegido, estableciéndose todos los mecanismos para su desarrollo por parte de las administraciones públicas. Este derecho, se justifica en el bienestar general basado en la necesidad de gozar de una calidad de vida que permita la participación equitativa y el beneficio de todos los resultados de cualquier investigación biomédica ${ }^{21}$. No obstante, como cualquier derecho, no es ilimitado sino que dispone de una serie de límites para evitar errores de la historia reciente donde la investigación biomédica ensayó numerosas terapias y técnicas de intervención que carecían de rigor, vulneraban los derechos humanos y se empleaban para propósitos alejados del bienestar social ${ }^{22}$.

Existen una serie de directrices éticas y legislativas en Bioderecho sobre las cuales se obtienen una serie de principios, de obligado cumplimiento, a considerar en cualquier investigación biomédica con personas humanas:

- La investigación constituye un derecho y deber del ámbito biomédico por lo que la libertad de investigación es un derecho fundamental. Este principio está recogido en el Código de Ética y Deontología Médica del Colegio Oficial de Médicos de España (CEDM); Carta de los Médicos de Hospital. Adoptada en Luxemburgo en abril de 1967 y revisada y adoptada en París en 1985. Organizaciones Médicas de las Comunidades Europeas (CMH1985); Declaración Universal sobre el Genoma Humano y los Derechos del Hombre. UNESCO, 11 de noviembre de 1997 (DUGH1997); Instrumento de Ratificación del Convenio para la Protección de los Derechos Humanos y la Dignidad del Ser Humano con respecto a las aplicaciones de la Biología y la Medicina (Convenio relativo a los derechos humanos y la biomedicina), hecho en Oviedo el 4 de abril de 1997 (BOE 251 de 20 de octubre de 1999 (IR1999); Declaración de Helsinki (DH1964); Directiva 2001/20/CE (D2001/20/CE); Convenio para la Protección de los Derechos Humanos y la Dignidad del Ser Humano con respecto a las aplicaciones de la biología y la medicina y Convenio relativo a los Derechos Humanos y la

Biología y la Medicina (Convenio relativo a los derechos humanos y la biomedicina), hecho en Oviedo el 4 de abril de 1997. BOE, núm. 251, de 20 de octubre de 1999, p. 36825 .

${ }^{21}$ AHUMADA CABANES, M., «La libertad de investigación científica, Orígenes de este derecho y configuración constitucional», Revista Estudios Socio-Jurídicos, vol. 10, núm. 1, enero-junio, 2008, pp. 11-49.

${ }^{22}$ CHUECA RODRÍGUEZ, R., "El derecho fundamental a la investigación científica», REDUR 6, diciembre 2008, pp. 5-15. 
Biomedicina de los estados de la Comunidad Europea, Oviedo 1997 (CPDH1997).

- Prioridad del ser humano sobre los beneficios y repercusiones de la investigación, debiéndose mantener la dignidad humana, ello implica una obligación de respeto a la integridad del ser humano y la dignidad de la persona. En cualquier investigación, los intereses de la ciencia y sociedad nunca podrán prevalecer sobre el bienestar del sujeto. Por tanto, deberán adoptarse las medidas para preservar la integridad física, psíquica y moral de las personas que participan como sujetos experimentales. Las referencias legislativas que contemplan este principio se recogen en DH1964, IR1999, CPDH1997, CEDM, Declaración de Nuremberg, 1967, Artículo 57-3 del Tratado de Roma (DN1967) y D2001/20/CE.

- Equidad entre los beneficios y riesgos asumidos en la investigación de manera que los riesgos de las personas participantes no sean superiores a los beneficios que obtiene la sociedad tal y como se expone en DH, IR1999, D2001/20/CE y Real Decreto $561 / 1993$ de 16 de abril por el que se establecen los requisitos para la realización de ensayos clínicos con medicamentos (RD561/1993) y CEDM.

- Participación voluntaria, libre e informada evitando vulnerar los derechos de las personas. Se debe asegurar que la participación en toda investigación y/ o experimento implicará el consentimiento libre e informado del participante después de recibir la información adecuada acerca de la naturaleza, finalidad del experimento, y los posibles riesgos o incomodidades que pueda implicar. Todo ello, según lo recogido en DH, CEDM, IR1999, D2001/20/CE, RD 561/1993.

- Garantizar el derecho a la intimidad de las personas participantes. Los investigadores tienen el deber de garantizar el derecho a la intimidad por ello, se guardará la más estricta confidencialidad de forma que no se viole la intimidad personal ni familiar de los sujetos participantes en la misma. Asimismo, deberán adoptarse las medidas apropiadas para evitar el acceso de personas no autorizadas a los datos (CEDM; DN1967; CMH1985; Postulado sobre el uso del ordenador en la Medicina. Basado en la Resolución Adoptada por la 27. ${ }^{\mathrm{a}}$ Asamblea Médica Mundial, Munich, Alemania 1973 y enmendado por la 35. ${ }^{a}$ Asamblea Médica Mundial, Venecia Italia 1983 (POUM); D2001/20/ CE; RD 561/1993 y Ley Orgánica 5/1992, de 29 de octubre, de 
Regulación del tratamiento automatizado de los datos de carácter personal (LO5/1992).

- Respeto a la dignidad e intimidad (CEDM; CMH1985; DUGH1997; IR1999; DH1964; D2001/20/CE; CPDH1997) de manera que toda investigación debe tratar a las personas participantes con el mayor respeto a su dignidad, creencias, intimidad y pudor.

- Protección para las personas más vulnerables que no puedan otorgar un consentimiento informado de manera libre, tales como, menores, ancianos, incapacitados, personas que sufran trastornos mentales, accidentados, enfermos, presos, o situaciones en las que se den especiales relaciones de autoridad. Este aspecto aparece detalladamente regulado en los artículos 6, 7, 9 y 17 del IR1999, así como en los considerados 3 y 4 de la D2001/20/CE, los artículos 4 y 5 de la D2001/20/CE y en el artículo 11 del RD561/1993.

- Prohibición del lucro y utilización de partes del cuerpo humano con fines fraudulentos, delictivos en el marco de cualquier investigación. En este sentido, cuando una parte del cuerpo humano haya sido extraída en el curso de una técnica de investigación, no podrá conservarse ni utilizarse con una finalidad distinta de aquélla para la que hubiera sido extraída, salvo de conformidad con los procedimientos de información y de consentimiento adecuados (IR1999).

- Protección del material genético humano Las directrices éticas y las leyes prohíben cualquier toda forma de discriminación de una persona a causa de su patrimonio genético. Sólo podrán hacerse pruebas genéticas predictivas de enfermedades genéticas y/o que permitan identificar al sujeto como portador de un gen responsable de una enfermedad, o detectar una predisposición o una susceptibilidad genética a una enfermedad, todo ello con fines médicos o de investigación médica (IR1999; DUGH1997).

\section{LEGISLACIÓN EN INVESTIGACIÓN BIOMÉDICA}

El ámbito legislativo se halla en constante evolución y supone una herramienta fundamental para establecer los límites de la investigación biomédica. Se describe, en el presente epígrafe, las principales normativas y declaraciones existentes en el ámbito internacional, europeo y español que deben ser respetadas. 


\section{IV.1 Legislación internacional}

Declaración Universal sobre el Genoma Humano y los Derechos Humanos de 1997

El principio de la dignidad, constituye la base fundamental para su interpretación y aplicación, ampliando el concepto de dignidad humana al reconocer el genoma humano como patrimonio de la humanidad. Entre los aspectos recogidos en la Declaración: a) No discriminar por causas genéticas; b) No alterar ni manipular el material genético; c) Precaución en un procedimiento, intervención y diagnóstico; d) Consentimiento previo, libre e informado a los fines de investigación, el tratamiento o el diagnóstico con el genoma humano; e) Confidencialidad de la información genética; f) Prohibición de prácticas contra la dignidad humana; g) Acceso irrestricto a los progresos de la biología, genética y medicina en materia de genoma humano, respetando la dignidad y derechos ${ }^{23}$.

Declaración Internacional sobre los Datos Genéticos Humanos de 2003

El texto de la declaración que la UNESCO aprobó, en el año 2003, se centra en los principios generales que deben establecerse sobre cuestiones que han generado una importante problemática en ámbito de la tecnología médica y genética. Se protegen una variedad de principios tales como el respeto de la dignidad de la persona, los derechos humanos, las libertades fundamentales, los principios de autonomía, libertad, igualdad, no-discriminación, justicia y solidaridad, así como de la protección de la vida privada y de la confidencialidad ${ }^{24}$.

Declaración Universal sobre Bioética y Derechos Humanos de 2005

Las características más destacadas de la Declaración incluyen: a) Establecimiento de un marco para el desarrollo de los derechos humanos; b) Los principios no pretenden resolver problemas bioéticos sino delimitar aquellos útiles para la Bioética en un consenso global,

${ }^{23}$ UNESCO, «Declaración Universal del Genoma Humanos y los Derechos Humanos», Rev Med Chile 1997; 125: 1485-9.

${ }^{24}$ UNESCO: Declaración Universal sobre el Genoma Humano y los Derechos Humanos, Paris: UNESCO, 1997. 
siendo la legislación interna de cada Estado la que deberá resolver cada caso especial ${ }^{25}$.

\section{Declaración de Helsinki 2008}

Esta declaración realiza varias regulaciones de interés para la investigación biomédica entre las que se destacan: a) Establecer un protocolo de la investigación que debe enviarse, para consideración, comentario, consejo y aprobación, a un comité de ética de investigación previo al inicio del estudio; b) El comité ético debe considerar las leyes y reglamentos vigentes en el país donde se realiza la investigación, como también las normas internacionales vigentes; c) El investigador tiene la obligación de proporcionar información al comité, en especial sobre todo incidente adverso grave ${ }^{26}$.

Guías Éticas Internacionales para la Investigación Biomédica en Seres Humanos (CIOMS, 2002)

La Organización Mundial de la Salud, en el año 2002, estableció una serie de Pautas Éticas Internacionales para la Investigación y Experimentación Biomédica en Seres Humanos que incluyen: Justificación ética y validez científica de la investigación biomédica en seres humanos; Comités de Evaluación Ética; Consentimiento informado; Incentivos para participar en una investigación; Beneficios y riesgos de participar en un estudio; Limitaciones especiales del riesgo con personas incapacitadas para otorgar consentimiento informado; Investigación con personas vulnerables, niños e individuos cuyos trastornos mentales o conductuales; Las mujeres como sujetos de investigación; Protección de la confidencialidad; Derecho a tratamiento y compensación de sujetos perjudicados; capacidad de evaluación ética y científica de la investigación biomédica ${ }^{27}$.

${ }^{25}$ GRACIA GUILLEM, D., «Investigación en sujetos humanos: implicancias lógicas, históricas y éticas», en Pautas éticas de investigación en sujetos humanos: nuevas perspectivas, Programa regional de Bioética OPS, OMS, 2003.

${ }^{26}$ Declaración de Helsinki. Principios éticos para las investigaciones con seres humanos. 59. ${ }^{a}$ Asamblea General, Seúl, Corea, octubre de 2008. Fecha de consulta: 20 de mayo de 2011]. Disponible en: http://www.wma.net/es/30publications/10policies/ b3/index.html.

${ }^{27}$ Council for International Organizations of Medical Sciences. International Ethical Guidelines for Biomedical Research Involving Human Subjects. Geneva: CIOMS; 2002: Preamble. 
Código de Nüremberg (1947) "Normas éticas acerca de la experimentación en seres humanos»

El Código se promulgó en 1947, como consecuencia de experimentos realizados durante la Segunda Guerra Mundial. Entre los objetivos del Código se incluyen proteger la integridad de las personas que se someten a un experimento, estipula las condiciones necesarias para la realización de trabajos de investigación en seres humanos, incidiendo en el consentimiento informado de esa persona ${ }^{28}$.

\section{IV.2 Legislación Europea}

\section{Convenio Europeo sobre Derechos Humanos y Biomedicina}

Este Convenio constituye el primer texto legal de ámbito europeo, con carácter vinculante para los estados miembros, que hace mención explícita a la investigación con humanos. Fue aprobado el 16 de noviembre de 1996 con el nombre abreviado de "Convenio Europeo sobre Derechos Humanos y Biomedicina», siendo ratificado por los Estados Miembros de la Unión Europea el 4 de abril de 1997 en Oviedo (España). Los contenidos del Convenio en materia de investigación con humanos, tienen como base un principio general de libertad de investigación en el ámbito de la biología y la medicina ${ }^{29}$.

Directiva 2001/20/CE sobre la Aplicación de Buenas Prácticas Clínicas en la Realización de Ensayos Clínicos de Medicamentos de Uso Humano

La Directiva 2001/20/CE del Parlamento Europeo y del Consejo, de 4 de abril de 2001, constituye una herramienta básica en la regulación de los ensayos clínicos de medicamentos de uso humano, como norma de carácter vinculante para todos los estados miembros de la Unión Europea. Se recogen los principios, ya establecidos en el Convenio Europeo sobre los requisitos para la realización de ensayos clí-

${ }^{28}$ DHAI, A., «The research ethics evolution: from Nuremberg to Helsinki. The research ethics evolution: from Nuremberg to Helsinki», S Afr Med J, 2014 Mar; 104 (3):178-80.

${ }^{29}$ INSTRUMENTO de Ratificación del Convenio para la protección de los derechos humanos y la dignidad del ser humano con respecto a las aplicaciones de la Biología y la Medicina (Convenio relativo a los derechos humanos y la biomedicina), hecho en Oviedo el 4 de abril de 1997. BOE núm. 251, de 20 de octubre de 1999, pp. 36825-36830. 
nicos con humanos. Todo ello, partiendo de la protección de los derechos fundamentales y la dignidad del ser humano. Un aspecto a destacar de esta Directiva es la regulación sobre una necesaria intervención por parte de un comité ético de investigación ${ }^{30}$.

\section{IV.3 Legislación Española}

Real Decreto Legislativo 1/2015, de 24 de julio

Este Decreto establece: 1. Definiciones de los conceptos necesarios para la comprensión del Decreto; 2. Protección de los individuos del ensayo, con especial regulación de los ensayos con menores y con personas incapacitadas; 3. Regula la figura de los Comités Éticos de Investigación Clínica (CEIC), estableciendo la creación del Centro Coordinador de CEIC con el objeto de facilitar el dictamen único que prevé la norma comunitaria; 4. Se establecen las normas de Buenas Prácticas Clínicas y la verificación de su cumplimiento; 5. Protección de los datos referentes a ensayos clínicos y su incorporación a las bases de datos nacionales y europea ${ }^{31}$.

\section{Ley de Investigación Biomédica 14/2007}

La Ley determinó que toda la investigación biomédica que se realice en España deberá cumplir con lo descrito en el texto legislativo. Los ámbitos sobre los cuales regula incluyen: a) Las investigaciones relacionadas con la salud humana que impliquen procedimientos invasivos; b) La donación y utilización de ovocitos, espermatozoides, preembriones, embriones y fetos humanos o de sus células, tejidos $\mathrm{u}$ órganos con fines de investigación y sus posibles aplicaciones clínicas; c) El tratamiento de aplicaciones biológicas; d) El almacenamiento y movimiento de muestras biológicas; e) Los biobancos ${ }^{32}$.

${ }^{30}$ Reglamento (UE) n. ${ }^{\circ}$ 536/2014 del Parlamento Europeo y del Consejo, de 16 de abril de 2014, sobre los ensayos clínicos de medicamentos de uso humano, y por el que se deroga la Directiva 2001/20/CE. Diario Oficial de la Unión Europea L 158/1, de 27 de mayo de 2014.

${ }^{31}$ Real Decreto Legislativo $1 / 2015$, de 24 de julio, por el que se aprueba el texto refundido de la Ley de garantías y uso racional de los medicamentos y productos sanitarios, BOE, núm. 177, de 25 de julio de 2015, pp. 62935-63030.

${ }^{32}$ Ley 14 /2007, de 3 de julio, de Investigación biomédica, BOE núm. 159, de 4 de julio de 2007, pp. 28826-28848. 


\section{LEY DE INVESTIGACIÓN BIOMÉDICA: LÍMITE EN EL ORDENAMIENTO JURÍDICO ESPAÑOL}

Analizando el Preámbulo de la Ley 14/2007, 3 de julio, de Investigación Biomédica, se manifiesta que uno de los ejes prioritarios es «asegurar el respeto y la protección de los derechos fundamentales y las libertades públicas del ser humano y de otros bienes jurídicos relacionados con ellos», todo ello, basado en la libre autonomía de la persona, de la cual se derivan los derechos específicos a otorgar el consentimiento e información previa que, desde el punto de vista de la acción investigadora ${ }^{33}$. La Ley pretende armonizar e integrar una serie de normas existentes en el ámbito nacional y europeo tales como el Convenio de Derechos Humanos y Biomedicina.

Previo a la Ley de Investigación Biomédica, el ordenamiento jurídico español disponía de principios y normativas que regulaban de manera directa e indirecta todo lo relacionado con la investigación, así aparecen recogidos en:

a) CE que se interrelaciona con la Ley 14/2007 y regula una parte del contenido esencial del derecho fundamental consagrado en el artículo 20 de la CE. En este sentido, el artículo 1.1 de la Ley, así lo establece al afirmar que su objeto es «regular, con pleno respeto a la dignidad e identidad humanas y a los derechos inherentes a la persona y la investigación biomédica» $\mathrm{y}$, en particular, las que en el mismo artículo se mencionan específicamente. También, el artículo 2 d) menciona que «la libertad de investigación y de producción científica en el ámbito de las ciencias biomédicas ${ }^{34}$. Por tanto, quedan definidos los derechos fundamentales, concretamente los relativos a libertad de producción científica, protección del respeto y dignidad de la persona.

b) Declaración Universal sobre Bioética y Derechos Humanos que considera a todos los seres humanos por igual, sin distinción alguna, y que deberían disfrutar de las mismas normas éticas elevadas en la investigación relativa a la medicina y ciencias de la vida ${ }^{35}$.

${ }^{33}$ Idem.

${ }^{34}$ Constitución Española de 1978, BOE de 29 de diciembre de 1978, pp. 29313-424.

${ }^{35}$ INSTRUMENTO de Ratificación del Convenio para la protección de los derechos humanos y la dignidad del ser humano con respecto a las aplicaciones de la Biología y la Medicina (Convenio relativo a los derechos humanos y la biomedicina), hecho en Oviedo el 4 de abril de 1997. BOE, núm. 251, de 20 de octubre de 1999, pp. 36825 a 36830 . 
c) Ley 14/2006, de 26 de mayo, sobre técnicas de reproducción humana asistida define el concepto de preembrión, definido como "grupo de células resultantes de la división progresiva del ovocito desde que es fecundado hasta 14 días más tarde». Además, en línea con lo que dispone la Constitución Europea, se prohíbe la clonación en seres humanos con fines reproductivos ${ }^{36}$.

d) Real Decreto Legislativo 1/2015, de 24 de julio, por el que se aprueba el texto refundido de la Ley de garantías y uso racional de los medicamentos y productos sanitarios. Este Decreto recoge las medidas contra productos falsificados y establece una integración de los cambios en la regulación de los productos sanitarios ${ }^{37}$.

e) Ley 41/2002, de 14 de noviembre, básica reguladora de la autonomía del paciente y de derechos y obligaciones en materia de información y documentación clínica aclara la situación jurídica, los derechos y obligaciones de los profesionales sanitarios, de la ciudadanía y de las instituciones sanitarias. Se ofrece información y la documentación clínica con las mismas garantías a toda la ciudadanía española, fortaleciendo con ello el derecho a la protección de la salud que reconoce la $\mathrm{CE}^{38}$.

f) Ley Orgánica 15/1999, de 13 de diciembre, de Protección de Datos de Carácter Personal que tiene por objeto garantizar y proteger, todo lo relativo al tratamiento de los datos personales, las libertades públicas y los derechos fundamentales de las personas físicas, especialmente de su honor e intimidad personal y familiar ${ }^{39}$.

En materia de investigación biomédica deben coexistir todas las garantías que permitan la libre producción y creación científica así como la libertad de investigación, pero su alcance no es ilimitado ya que se requiere una autorización previa para la realización de determinadas investigaciones reguladas por los Comités de Bioética.

${ }^{36}$ Ley 14/2006, de 26 de mayo, sobre técnicas de reproducción humana asistida, BOE, núm. 126, de 27 de mayo de 2006, pp. 19947-19956.

${ }^{37}$ Real Decreto Legislativo $1 / 2015$, de 24 de julio, por el que se aprueba el texto refundido de la Ley de garantías y uso racional de los medicamentos y productos sanitarios, BOE, núm. 177, de 25 de julio de 2015, pp. 62935-63030.

${ }^{38}$ Ley $41 / 2002$, de 14 de noviembre, básica reguladora de la autonomía del paciente y de derechos y obligaciones en materia de información y documentación clínica, BOE, núm, 274, pp. 40126-40132.

${ }^{39}$ Ley Orgánica 15/1999, de 13 de diciembre, de Protección de Datos de Carácter Personal, BOE, núm. 298, de 14 de diciembre de 1999, pp. 43088-43099. 
Por otro lado, los límites establecidos en el apartado 4 del artículo 20 de la CE determinan que todos los derechos constitucionales pueden tener límites ${ }^{40}$. El Tribunal Constitucional (STC 2/1982, de 29 de enero $)^{41}$ determinó que no existe en el ordenamiento español «derechos ilimitados y que todo derecho tiene sus límites» ${ }^{42}$ manifestación que ha sido repetida en numerosas sentencias del Alto Tribunal. Esta idea de limitación de los derechos, evita que el reconocimiento de un derecho provoque una situación de abuso o de uso antisocial del mismo ${ }^{43}$.

Por todo ello, debe interpretarse el alcance de la investigación biomédica como un derecho que faculta a los investigadores y a los profesionales del campo de la biomedicina para el ejercicio libre de la misma y a las personas participantes para participar en ella, siempre que todo se desarrolle en el estricto marco de los derechos y libertades fundamentales establecidos en la $\mathrm{CE}$ y del resto del ordenamiento jurídico ${ }^{44}$.

\section{CONCLUSIONES}

El presente trabajo enmarca los límites que condicionan y determinan la investigación biomédica. El inexorable desarrollo de la medicina y las nuevas tecnologías aplicadas a la biomedicina implica la participación directa del ámbito jurídico. En este sentido, se constata la relación entre legislación española en contraposición con la legislación derivada. Por ello, se utilizan como elementos básicos del análisis, los aspectos relativos a los derechos fundamentales de la persona. En esta línea, la legislación existente se justifica en base a varios aspectos fundamentales tales como la dignidad de la persona, el consentimiento informado, la intimidad personal y la libertad personal.

El análisis sobre la legislación en materia de investigación biomédica debe realizarse de conformidad con los principios constitucionales, procedentes de aquellos otros principios y valores que se recogen en el ordenamiento jurídico internacional. El derecho a

${ }^{40}$ GÓMEZ, Y., Derecho Constitucional Europeo: derechos y libertades, Ed. Sanz y Torres, 2005, pp. 250-275.

${ }^{41}$ STC 2/1982, de 29 de enero. BOE núm. 63, de 14 de marzo de 2009. Págs. 51-58.

${ }^{42}$ CHUECA RODRÍGUEZ, R., "El derecho fundamental a la investigación científica», REDUR 6, diciembre 2008, pp. 5-15.

${ }^{43}$ Código Civil Español. BOE. núm. 206, de 25/07/1889.

${ }^{44}$ SÁNCHEZ CARO, J., Investigación Biomédica en España: aspectos bioéticos, jurídicos y científicos, 2007, Editorial Comares, pp. 14-16. 
participar en una investigación biomédica se enmarca en la interpretación de la libertad como derecho-autonomía y muestra una vertiente positiva que permite decidir libremente sobre su propia participación y, una vertiente negativa, que protege igualmente su decisión de no participar.

El derecho a la investigación biomédica permite analizar y regular nuevos conflictos en torno a las técnicas de investigación que se puedan implantar. Así, se debe extender el ámbito de la libertad hasta donde sea posible sin vulnerar otros valores y principios constitucionalmente reconocidos. 
